\title{
Noise Spectra for Monolithic Detector Arrays
}

\author{
MITCHELL W. FINKEL, LESLIE L. THOMPSON, AND ROBERT J. SULLIVAN
}

\begin{abstract}
A brief review of nonstationary processes is extended so as to include mixed stationary processes as they apply to the determination of the power density spectrum for monolithic detector arrays. An analog method for determining noise spectra is developed. Both theoretical and experimental results are presented.
\end{abstract}

\section{INTRODUCTION}

W ITH THE RAPID maturation of large-scale integration (LSI), hundreds, even thousands, of detectors are now contained within a single monolithic device. Evaluating the performance of these devices requires that we review some of the measurement procedures. Certainly the noise power density spectrum is one such fundamental measurement. The usual noise model is something of a compromise between simplicity and fidelity. A more realistic model would not only consider the contributions made by each noise generator, but also because of the distributed nature of these sources, the model would consider the associated transformations. For example, the transformation associated with correlated sampling which effectively suppresses low-frequency (LF) noise is graphically illustrated by the power density spectrum.

Thus the power spectrum affords us a more informed view of a noise process than is revealed by merely considering the moments of some presumed distribution (e.g., the mean and variance). Indeed, if we begin with the presumption that we are dealing with a normal process, then the entire statistical structure is revealed by the spectrum. In addition, there is the bonus that even a cursory reading of the spectrum should help distinguish between intraneous and extraneous noise.

Perhaps we should say something about the nature of the output of monolithic detector arrays. Typically, signals are processed in the analog domain, and after passing through a sample-and-hold (SH) circuit, the final video output is provided for display or digital conversion. Since a great many detectors are sequentially read out through a single video channel, what one observes is a contiguous stream of nearly rectangular pulses of constant width but varying amplitudes (similar to pulse amplitude modulation). It is these varying amplitudes that abound with information about both the response by each detector to the incident irradiance and the associated noise. Our primary concern will be the extraction of this information, particularly as it relates to the noise content.

We will begin with an abbreviated discussion of spectra

Manuscript received March 6, 1978.

The authors are with NASA/Goddard Space Flight Center, Greenbelt, MD 20771. for nonstationary processes, since in general we should anticipate a nonstationary noise process. Initially, we will essentially follow the Bendat-Piersol [1] exposition. Subsequently, we will develop a rather direct method for realizing the noise power spectrum. Although the measurements contained in the following sections pertain to a 96-element Westinghouse photodiode linear array, the method outlined should be applicable to any monolithic device.

\section{Nonstationary Processes}

The spectra of special interest, the time-averaged power spectrum, can be expressed as

$$
G(f, T)=\frac{1}{T} \int_{T} \frac{1}{\Delta f N} \sum_{i=1}^{N} x_{i}^{2}(t, f, \Delta f) d t
$$

where the $x_{i}$ 's are sample functions forming a random noise process, $f$ is the center frequency, $\Delta f$ is the bandwidth of definition about the center frequency, and $T$ is the time interval over which the spectrum is averaged. Suppose we follow the steps implicit in this expression: 1) filtering, 2) squaring, 3) ensemble averaging, and 4) time averaging. We can begin with a sample function, $x_{i}(t)$, which is a member of the ensemble and consider the input-output relationship of a filter as given by the convolution integral,

$$
x_{i}(t, f, \Delta f)=\int_{-\infty}^{\infty} h(\xi) x_{i}(t-\xi) d \xi .
$$

For an ideal bandpass filter whose frequency response $H(v)$ is unity within the bandpass and zero elsewhere, a transform exists such that,

$$
\begin{aligned}
h(\xi) & =\int_{-\infty}^{\infty} H(v) \exp (2 \pi j \xi v) d v \\
& =\frac{\sin (\pi \Delta f \xi)}{\pi \xi} \cos 2 \pi f \xi .
\end{aligned}
$$

Now squaring,

$$
x_{i}^{2}(t, f, \Delta f)=\int_{-\infty}^{\infty} h(\xi) h(\eta) x_{i}(t-\xi) x_{i}(t-\eta) d \xi d \eta .
$$

Ensemble averaging and dividing by the bandpass leads to time-varying power spectrum,

$$
G(t, f)=\frac{1}{\Delta f} \iint_{-\infty}^{\infty} h(\xi) h(\eta) \phi(t-\xi, t-\eta) d \xi d \eta
$$


where $\phi(t-\xi, t-\eta)=\phi\left(t_{1}, t_{2}\right)$ is the autocorrelation function of $x(t)$. We can remove the time dependence by averaging the autocorrelation function. But first let us introduce a new set of variables

$$
\begin{aligned}
t^{\prime} & =t-\xi \\
\tau & =\xi-\eta
\end{aligned}
$$

so that the time-averaged autocorrelation function can now be written as

$$
\frac{1}{T} \int_{0}^{T-\tau} \phi\left(t^{\prime}, t^{\prime}+\tau\right) d t^{\prime}=\langle\phi(\tau, T)\rangle .
$$

Inserting (3) and (6) into (5), we have

$$
\begin{aligned}
G(f, T)=\frac{1}{\Delta f} \int_{\infty}^{\infty} \int_{\infty}\langle\phi(\tau, T)\rangle \exp (-2 \pi j f \tau) \\
\cdot \frac{\sin \pi \Delta f \eta \sin \pi \Delta f(\tau+\eta)}{\pi^{2} \eta(\tau+\eta)} d \tau d \eta .
\end{aligned}
$$

Integrating with respect to $\eta$ yields [2]

$$
\frac{1}{\Delta f} \int_{-\infty}^{\infty} \frac{\sin \pi \Delta f \eta \sin \pi \Delta f(\tau+\eta)}{\pi^{2} \eta(\tau+\eta)} d \eta=\operatorname{sinc} \pi \Delta f \tau
$$

So we can now say,

$$
G(f, T)=\int_{-\infty}^{\infty}\langle\phi(\tau, T)\rangle(\operatorname{sinc} \pi \Delta f \tau)[\exp (-2 \pi j f \tau)] d \tau
$$

The time-averaged power spectrum (9) submits to several possible interpretations. Turning to the convolution theorem, for example, we interpret (9) as a running mean. That is, multiplying the autocorrelation function by the sinc function in the transform domain corresponds to the convolution of $G(f)$ with the rectangle function $\Pi(f)$ in the frequency domain. Or to put it another way, the weighting function, sinc $\pi \Delta f \tau$ can be viewed as a kind of sliding scale factor which continuously renormalizes the instantaneous power spectrum so that, effectively, it becomes locally stationary. We also note that in the limit as $T \rightarrow \infty$ and consequently $\Delta f \rightarrow 0$, equation (9) reduces to the more familiar representation

$$
G(f)=2 \int_{-\infty}^{\infty} \phi(\tau) \exp (-2 \pi j f \tau) d \tau
$$

We conclude that once the appropriate weighting function is introduced, determining the noise power spectrum reduces to the familiar Weiner relationship.

\section{NoISE SPECTRA}

Our treatment thus far has been quite general. Again, we consider the typical output of a linear array. (Note: The following can be easily interpreted to apply to frame imaging devices as well.) Fig. 1 is meant to be a representation of such a video output. As the figure suggests, each detector element is read out sequentially. At the end of the line time $T$, the

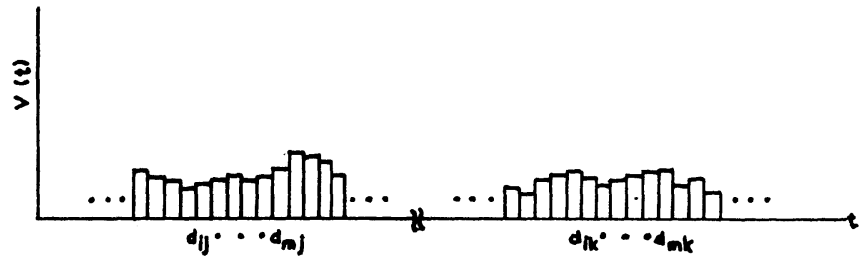

Fig. 1. An idealized representation of an output of a linear array showing two consecutive sequences of rectangular pulses with constant widths $\zeta$ and random heights. Each detector element is tagged with a double subscript. The first subscript identifies the element number and the second refers to the sequential order.

sequence is once again initiated and another video line is read out. The process repeats until the end of the data collection/imaging frame. In the figure, each detector element is tagged with a double subscript. The first subscript identifies the element number and the second refers to the sequential order. Now suppose we select some detector, ignoring for the moment all others, and sequentially display its output signal line after line. What one would see is a series of rectangular pulses of identical widths but varying amplitudes depending upon the instantaneous signal and noise level. We can represent this series by [3],

$$
f(t)=\sum_{N} X(t) \Pi\left(\frac{t-b_{N}}{\zeta}\right)
$$

where $X(t)$ refers to the random heights and $\Pi(t)$ represents the rectangular function with displacement $b_{N}$ and widths $\zeta$. Since $f(t)$ is a random function, the spectrum must be determined according to the Weiner theorem by transforming the autocorrelation function. Autocorrelating $f(t)$, we find that

$$
\phi\left(t_{1}, t_{2}\right)=\phi_{x}\left(t_{1}, t_{2}\right) \sum_{N} \Pi\left(\frac{t_{1}-b_{N}}{\zeta}\right) \cdot \sum_{M} \Pi\left(\frac{t_{2}-b_{M}}{\zeta}\right)
$$

where $\phi_{x}\left(t_{1}, t_{2}\right)$ is the autocorrelation function for the random part of $f(t)$. We note that the series multiplication is prescribed according to $t_{2}=t_{1}+\tau$ and that the rectangle functions are centered on $b_{N}$ and $b_{M}$ at $t_{1}$ and $t_{2}$, respectively. In other words, the correlation must reflect the allowed displacements. But the autocorrelation of a rectangle function can also be expressed in terms of the triangle function. Hence,

$$
\sum_{N} \Pi\left(\frac{t-b_{N}}{\zeta}\right) \cdot \sum_{M}\left(\frac{t+\tau-b_{M}}{\zeta}\right)=\sum_{N} \Lambda\left(\frac{\tau-b_{N}}{\zeta}\right)
$$

where $\Lambda(\tau)$ is the triangle function. Introducing the replicating function $\Pi \|(\tau)$, equation (13) can also be written as,

$$
\phi_{\Pi}(\tau)=\sum_{N} \Lambda\left(\frac{\tau-b_{N}}{\zeta}\right)=\Lambda\left(\frac{\tau}{\zeta}\right) * \frac{1}{T} \pi\left(\frac{\tau}{T}\right)
$$

where $*$ denotes convolution. Now, according to the Weiner theorem, the spectrum is given by,

$$
S(f)=\int_{-\infty}^{\infty} \phi_{x}(\tau) \cdot \phi_{\Pi}(\tau) \cos 2 \pi f \tau d \tau
$$


where we have tacitly assumed a stationary process, so that

$$
\phi_{x}\left(t_{1}, t_{2}\right)=\phi_{x}(\tau) .
$$

Then by the convolution theorem we need to determine,

$$
F\left[\phi_{x}(\tau)\right] * F\left[\phi_{\Pi}(\tau)\right]
$$

where $F$ signifies the Fourier transform. Let,

$$
F\left[\phi_{x}(\tau)\right]=G(f)
$$

and again referring to the convolution theorem,

$$
\begin{aligned}
F\left[\phi_{\Pi}(\tau)\right] & =F\left[\Lambda\left(\frac{\tau}{\zeta}\right) * \frac{1}{T} \pi\left(\frac{\tau}{T}\right)\right] \\
& =F\left[\Lambda\left(\frac{\tau}{\zeta}\right)\right] \cdot F\left[\frac{1}{T} \Pi\left(\frac{\tau}{T}\right)\right]
\end{aligned}
$$

By the similarity theorem,

$$
F[\Lambda(\tau / \zeta)]=\zeta \operatorname{sinc}^{2} \zeta f
$$

and

$$
F\left[\frac{1}{T} \Pi(\tau / T)\right]=\Pi(T f)
$$

But

$$
\Pi(T f)=\frac{1}{T} \sum_{N} \delta(f-N / T)
$$

So that

$$
S(f)=\frac{\zeta}{T} \sum_{N} G(f-N / T) \operatorname{sinc}^{2} \zeta N / T .
$$

So we see that $G(f)$ will be replicated on a set of impulses whose amplitudes are determined by the sinc function. We could isolate $G(f)$ and thereby separate the periodic and random components of the process by multiplying $S(f)$ by an appropriate rectangle function, which will separate the first period from the rest of the periodic train. It is of some interest to just briefly compare (9) and (21). To begin with, the assumption that the process is stationary would remove the sinc function in (9). In the previous section we assumed a continuous random process, whereas here we are concerned with a mixed process that describes the discrete output signals of a linear array due to random noise. Hence, the appearance of the multiplicative autocorrelation function and spectrum as described by (21). Thus, the two expressions [equations (9) and (21)] are, in fact, quite analogous.

\section{MEASUREMENTS}

Given some random function, we can realize the power density spectrum by either analog or digital methods. The method is optional; the results should be equivalent. If we choose, as we have, the analog option, we will obviate the need for autocorrelating and transforming as necessitated by digital methods. That is to say, we will accept the rms voltage output of a wave analyzer (H.P. $3581 \mathrm{~A} / \mathrm{C}$ ) as the essential beginning of the operation to determine the power density spectrum. If we then divide that output by the square root of the analyzer's selected bandpass, we will have

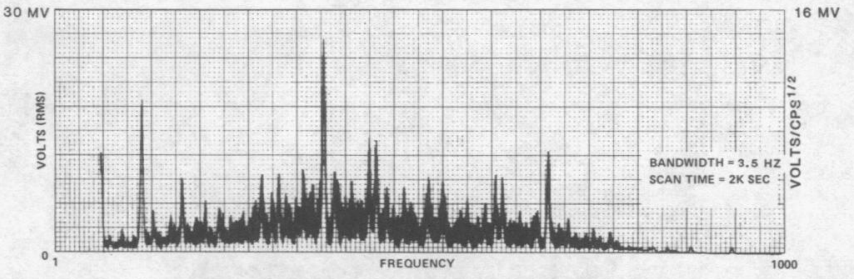

Fig. 2. The rms noise voltage and the corresponding root power spectrum are given as a function of frequency. The bandwidth is the calculated equivalent noise bandwidth. The $2 \mathrm{~K}$ seconds refers to the sweep time of the analyzer for a record length of 10 seconds.

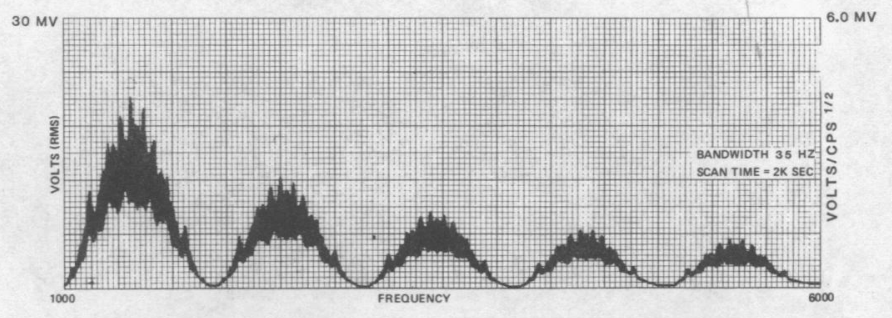

Fig. 3. The replication and the envelope formed by the sinc function for part of the periodic train as predicted by (21).

determined the root power spectrum whose units are $\mathrm{V} / \mathrm{Hz}^{1 / 2}$. Since the units of the power density spectrum are $\mathrm{V}^{2} / \mathrm{Hz}$, a further squaring operation is indicated. Here again we have two options. We can either pass the output of the wave analyzer through a squaring circuit and then divide by the bandpass, or simply rescale the ordinate so that we can read directly in terms of power. For most applications the root power spectrum suffices. Consequently, it is the spectrum usually reported in the literature.

With all this in mind, we return to (11) and Fig. 1 and agree to synchronously sample the pulse amplitudes $d_{i j}$, $d_{i k}, \ldots, d_{i n}$, holding each value until the next signal amplitude appears. Clearly, holding $X(t)$ for a period $T$ and multiplying by $\Pi(t)$ results in an equivalent $f(t)$, since $\Pi(t)$ would obliterate $X(t)$ outside the interval $\zeta$. One further point, the particular form of $\Pi(t)$ is of no special consequence since it relates to the periodic component. With this in mind we have found it expedient in our case to collect nominally 10000 samples of digitized data from a given element in computer memory. The contents of the computer memory are sequentially cycled through a digital-to-analog converter. The resulting analog signal is continuously cycled through a wave analyzer. Alternatively, the analog output of the array could have been passed through a SH circuit which is keyed to the time position of a given detector element and could have gone directly to the wave analyzer [4].

The results as predicted by (21) are shown in Fig. 2 and 3 . It is quite clear from the figure that the noise in the LF region is suppressed. This very significant development is attributable to the double sampling signal processing [5] on the detector chip which effectively transforms an inherently nonstationary process to a stationary process. There is also further evidence gained by an independent statistical study which was concerned with the mean and the variance. This study supports the contention that the process is indeed stationary. The spectrum illustrated in Fig. 2 is, of course, the root power spectrum. The main intent of exhibiting Fig. 

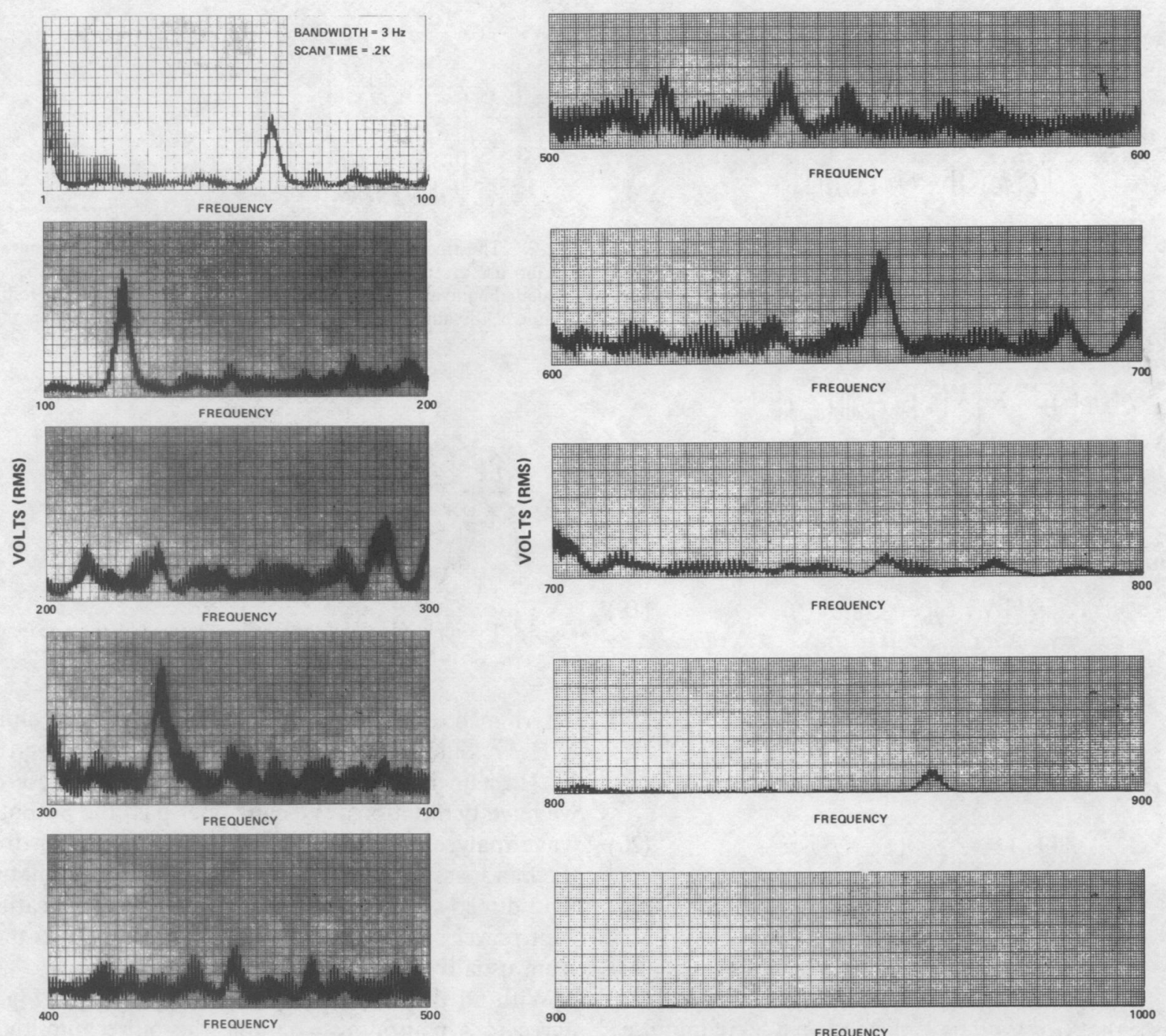

Fig. 4. An expanded view of the noise power spectrum. The extraneous 60 and $120 \mathrm{~Hz}$ pickup is clearly evident. Note the analyzer's frequency range begins at $15 \mathrm{~Hz}$.

3 is to show the replication resulting from the periodicity, and the envelope formed by the sinc function. Fig. 4 offers a more detailed presentation of the spectrum for the indicated frequency range. We note the ubiquitous $60 \mathrm{~Hz}$ peak as well as some of the higher harmonics.

There are two relatively easy checks we can make to validate our measurements. First of all, it is a simple matter to cycle the analog signal through a true rms voltmeter. Consistently, we have found excellent agreement between the rms voltage readings and statistical determinations of the same quantity. We know, therefore, that the input to the wave analyzer is a proper representation of the original signal. It is also possible to validate the output. We recall that the area enclosed by the power spectrum is equal to the variance. To put it succinctly,

$$
\sigma^{2}=\int_{0+}^{\infty} G(f) d f
$$

where the notation $0+$ means that the integration begins just past the edge of the dc component. Here again, even a rather elementary numerical integration of the noise power spectrum is in very reasonable agreement with a statistical determination of the variance.

\section{CONCLUSIONS}

Essentially we have shown that once the output of a monolithic detector array has passed through a sample and hold circuit, determining the noise spectra reduces to the more familiar problem of measuring spectra of an elementary detector. We have also shown that the resultant spectra is multiplicative, and is replicated on a periodic train whose periodicity is determined by the line time.

\section{REFERENCES}

[1] J. S. Bendat and A. G. Piersol, Measurement and Analysis of Random Data. New York: Wiley, 1966.

[2] F. Donati, "Finite-time averaged power spectra," IEEE Trans. Inform. Theory, vol. 17, pp. 7-16, Jan. 1971.

[3] R. N. Bracewell, The Fourier Transform and It's Applications. New York: McGraw-Hill, 1965.

[4] M. W. Finkel, R. J. Sullivan, and L. L. Thompson, "Procedures for the characterization of linear arrays," NASA/Goddard Space Flight Center X-Document, X-942-77-75, Apr. 1977.

[5] M. H. White, D. R. Lampe, F. C. Blaha, and I. A. Mack, "Characterization of surface channel CCD image arrays at low light levels," IEEE J. Solid-State Circuits, vol. SC-9, pp. 1-13, Feb. 1974. 